\title{
Raft.1, A Monoclonal Antibody Raised Against the Raft Microdomain, Recognizes G-Protein $\beta 1$ and 2, Which Assemble Near Nucleus After Shiga Toxin Binding to Human Renal Cell Line
}

\author{
Yohko U. Katagiri, Kazuhiro Ohmi, Weiran Tang, Hisami Takenouchi, \\ Tomoko Taguchi, Nobutaka Kiyokawa, and Junichiro Fujimoto \\ Department of Developmental Biology, National Research Institute for Child Health and Development, Tokyo, Japan
}

SUMMARY: Raft microdomains are glycolipid-enriched microdomain scaffolding molecules involved in signal transduction. The binding of Shiga toxin to globotriaosyl ceramide in raft microdomains of the human renal tubular cell line ACHN causes temporal activation of Src-kinase Yes. To study the downstream signaling mechanism proceeding to the activation of Yes, we raised monoclonal antibodies (MAbs) against raft microdomains. The MAbs were screened on the basis of, first, binding to raft microdomains with dot-blot immunostaining, second, intracellular localization of the epitope by flowcytometry after permeabilization, and third, translocation of the antigen molecules after Stx treatment by immunohistochemical staining. Raft. $1 \mathrm{MAb}$ bound to the molecules that accumulated to the particular region near the nucleus after Stx treatment. Two-dimensional Western blotting and matrix-assisted laser desorption/ionization time of flight mass spectrometry analysis revealed that the antigen molecule is GTP binding protein $\beta$ subunits 1 and 2 (G $\beta 1$ and 2). That Raft.1 recognized $\mathrm{G} \beta 1$ and 2 was further confirmed by the reactivity to recombinant $\mathrm{G} \beta 1$ and 2 proteins. To our knowledge, this is the first report of production of a MAb recognizing $\mathrm{G} \beta 1$ and 2 . Because $\mathrm{G} \beta 1$ and 2 are highly conserved all through organisms and are deeply involved in signal transduction, Raft. 1 is expected to be utilized frequently in research. (Lab Invest 2002, 82:1735-1745).

$P^{1}$ lasmalemma microdomains that are rich in glycosphingolipids (GSL) and cholesterol are often called raft (Simons and Ikonen, 1997). Recent studies on raft signaling microdomains have progressed in various fields, and our knowledge of their characteristics and cellular functions has been accumulating. Rafts are lateral assemblies of GSL and cholesterol that associate with specific proteins, and they are thought to be platforms serving as scaffolds to facilitate the apical sorting or association of signaling molecules, increase the rate of interactions, and enhance crosstalk networks.

CD77, namely Gb3 (globotriaosyl ceramide) expressed on the cell surface, serves as a receptor for Shiga toxin (Stx), an enterotoxin produced by Shigella

DOI: 10.1097/01.LAB.0000044134.23951.C8

Received September 30, 2002.

This work was supported in part by a Grant for Pediatric Research (12C-01) from the Ministry of Health and Welfare of Japan. It was also supported by a grant from the Japan Health Sciences Foundation for Research on Health Sciences Focusing on Drug Innovation. Additional support was provided by the Program of the Research and Development Promotion Division, Science and Technology Promotion Bureau, STA for Organized Research Combination System.

Address reprint requests to: Dr. Junichiro Fujimoto, Department of Developmental Biology, National Research Institute for Child Health and Development, 3-35-31 Taishido, Setagaya-Ku, Tokyo 154-8567, Japan. E-mail:jfujimoto@nch.go.jp dysenteriae serotype 1 and enterohemorrhagic Escherichia coli. Stx consists of an A subunit, which is responsible for inducing cell death, and a pentamer of $B$ subunits, that acts as a receptor-binding site but that lacks any toxicity. Stx bound to $\mathrm{Gb3}$ is readily internalized and causes cell death in various CD77expressing cells (Lingwood, 1996). Although the B subunit has no toxicity, a series of studies indicates that it alone participates in transducing cell signaling and induces apoptosis in some cells such as in Burkitt's lymphoma (Taga et al, 1997). We have already demonstrated that binding of Stx to $\mathrm{Gb3}$ in the detergent-insoluble microdomains (DIM)/rafts of ACHN cells causes temporal activation of the Srcfamily kinase Yes (Katagiri et al, 1999). To study the downstream signaling mechanism proceeding to the activation of Yes, it is critically important to characterize the constituent molecules in these microdomains.

In order to examine the signaling mechanism of Stx binding in rafts, we raised MAbs against the raft microdomains of ACHN cells. Raft microdomains were obtained as detergent-insoluble, low-density microdomains by sucrose density-gradient centrifugation of $1 \%$ Triton lysate. We have already reported that simple subcutaneous injection of a DIM suspension can elevate the serum titer after several boosts and that every clone reacting to the lipid fraction of DIM recognizes monosialosyl galactosylgloboside (MSGG), which carries the stage-specific 
embryonic antigen-4 (Katagiri et al, 2001). The dotblot immunostaining assay used for the first screening is a time- and reagent-saving method. We further showed that some clones were reactive only with permeabilized $\mathrm{ACHN}$ cells, indicating that these antigens or epitopes reside on the cytoplasmic side of the raft. It is therefore highly probable that these molecules are deeply involved in signal transduction via raft microdomains.

In the present study, we describe a raft-reacting MAb, Raft.1, that binds to ACHN cells only after exposure to a permeabilizing reagent. Further, confocal microscopic observation showed that the antigen molecules accumulated near the nucleus after a 30minute culture in the presence of Stx. Twodimensional (2-D) Western blotting and mass spectrometric analysis after 2-D separation revealed that Raft. 1 recognizes GTP binding protein $\beta$ subunits 1 and 2 (G $\beta 1$ and 2), known as transducin 1 and 2, respectively. Because $\mathrm{G} \beta$ is widely distributed and acts as a modulator or a transducer in various transmembrane signaling systems, Raft. 1 binds not only to $\mathrm{ACHN}$ cells but also to a variety of other types of cells, such as leukocytes. We also describe the immunostaining pattern of frozen sections of rat tissues and propose that Raft.1 MAb is a useful tool for signal transduction analysis as well as histochemical analysis of $\mathrm{G} \beta$ localization.

\section{Results}

\section{Production of MAbs}

Using dot-blot immunostaining and flowcytometric analysis, we screened out three clones that reacted to permeabilized ACHN cells from hybridomas producing MAbs against ACHN raft microdomains (data not shown). We further screened a clone of Raft.1, of which the immunohistochemical staining pattern changed after 30 minutes of Stx stimulation. Raft.1 stained plasma membrane of unstimulated ACHN cells, while the particular area near the nucleus was heavily stained in Stx-stimulated ACHN cells (Fig. 1a). The other clones such as Raft.2 that recognize MSGG, a major sialylated GSL of $A C H N$ raft, stained $A C H N$ cells, with no difference between Stx stimulation and no stimulation (Fig. 1b). Phase microscopic observation showed that Stx stimulation under this condition did not induce any morphologic changes in ACHN cells. The Raft. 1 was subtyped as mouse $\operatorname{lgM}, \kappa$ immunoglobulin.

\section{Flow Cytometric Analysis}

Nonpermeabilized ACHN cells stained with Raft.2 but not with Raft.1 or anti-Yes antibody. Permeabilized ACHN cells, however, positively stained with both of the latter (Fig. 2a). Because Src-kinase Yes is well known to be anchored to the cytoplasmic side of the plasma membrane and is resistant to detergent solubilization, it appeared likely that the Raft.1-defined molecule is also resistant to detergent and that the epitope is localized within the cell. Lymphoid cells

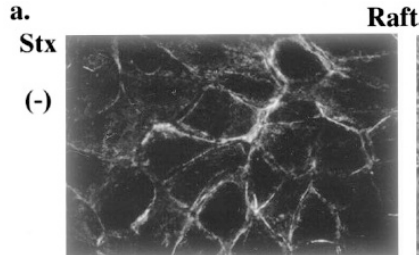

Raft.1

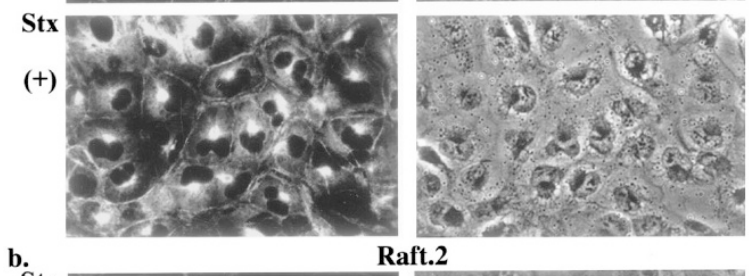

b.
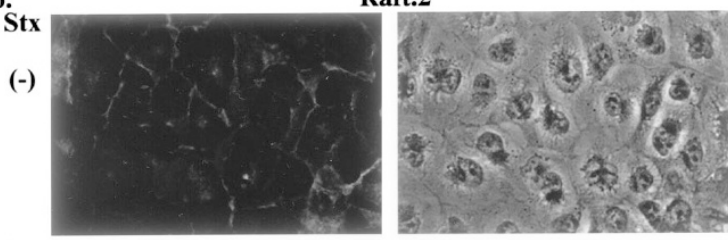

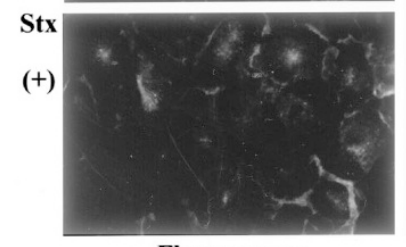

Fluorescence

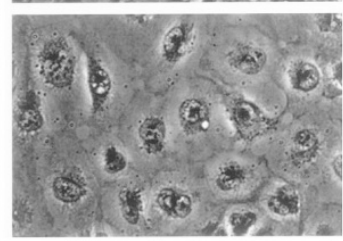

Phase Contrast $\overline{50 \mu \mathrm{m}}$
Figure 1.

Translocation of Raft.1-defined antigens after Stx stimulation. ACHN cells grown in RS-coated chamber slides were cultured in the absence $(-)$ or the presence $(+)$ of Stx for 30 minutes and fixed as detailed under "Materials and Methods." The slides were observed with fluorescence microscope (left) and phase contrast microscope (right).

were also examined, and the peripheral leukocytes prepared from the human, pig, rat (Fig. 2b), and all human B cell lines tested (Fig. 2c) stained well with Raft.1. The Raft.1 antigen was shown to be expressed at all stages, from pro-B cell to lgG-secreting cells, as judged from various human lines.

\section{Antigen Analysis by 2-D Western Blotting}

Two-dimensional Western blotting analysis was performed to determine the biochemical nature of the Raft.1-defined molecule. The raft protein $(6 \mu \mathrm{g})$ was separated into more than 100 spots by 2-D PAGE (Fig. 3a). Raft. 1 MAb specifically bound to the spot whose isoelectric point was slightly more basic than actin, and it had an apparent molecular weight of approximately 34 k (Fig. 3b). Because N-terminal determination of the spot cut from the Coomassie Brilliant Blue (CBB)-stained blot failed because of the $\mathrm{N}$-acetylation block, we carried out matrix-assisted laser desorption/ ionization time-of-flight mass spectrometry (MALDITOF MS) analysis on the three spots, as indicated in Figure 3c. Accordingly, the three circles marked by the arrowhead, "A," and "blank" in Figure 3c were cut out of the CBB-stained gel for in-gel digestion with trypsin and were analyzed with MALDI-TOF MS. 
a.ACHN cells

unpermeabilized permeabilized

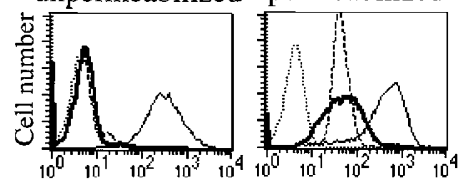

b.Peripheral leukocytes
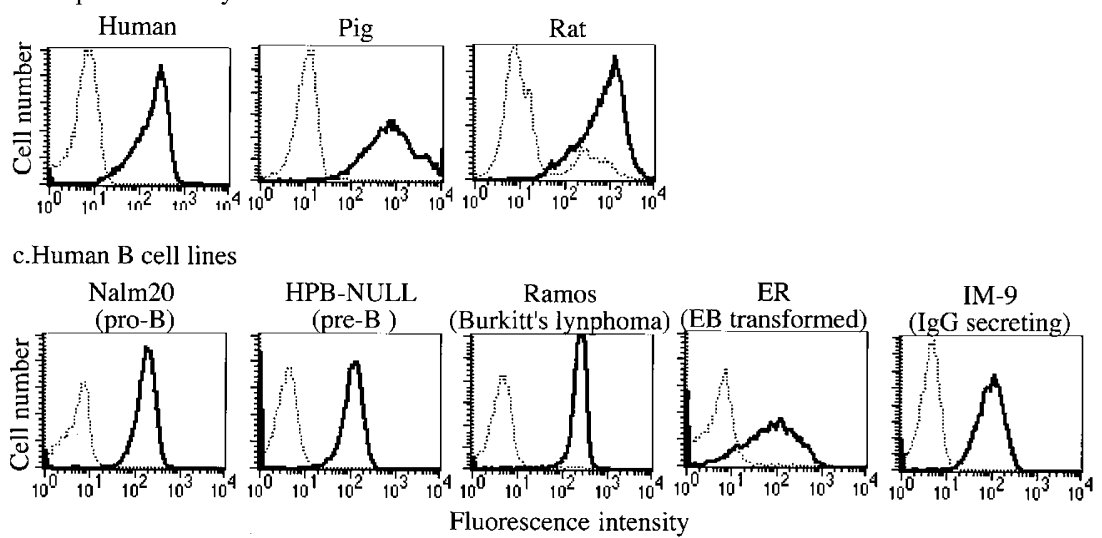

\section{Figure 2.}

Flowcytometric analysis of Raft.1 antigen expression. a, ACHN cells were stained with control antibody (…...), Raft.1 (bold -), Raft.2 (-), and anti-Yes MAb ( - ) before and after permeabilization. b. Peripheral leukocytes obtained from a human, pig, and rat were stained with control antibody (…....) and Raft.1 (bold -) after permeabilization. c, Cultured B cell lines of various stages from the pro-B cell stage to the Ig-secreting stage were stained with Raft.1 as in b.

\section{Identification of the Antigen Molecule by MALDI-TOF MS}

Eleven possible peptide signals with masses $(\mathrm{m} / \mathrm{z})$ of 902.4657, 916.4815, 1009.5253, 1352.6146, $1551.7077,1577.7226,1728.8775,1770.9025$, 1945.9613, 2074.0577, and 2158.0942 were detected (Fig. 4), and a search of the resulting peptide masses with MS-Fit resulted in selection of two entries, $\mathrm{G} \beta 1$ and $\mathrm{G} \beta 2$ (Table 1). ' ${ }^{1}$ Serine was blocked by $\mathrm{N}$-acetylation. The $1728.9(\mathrm{~m} / \mathrm{z})$ fragment was further confirmed to be $\mathrm{N}$-actylated SELDQLRQEAEQLK by postsource decay MS/MS. G $\beta 1$ and $\mathrm{G} \beta 2$ have molecular sizes of $37,376.59$ and $37,330.69$, respectively, and their isoelectric points are 5.55. GTP binding protein is a heterotrimer composed of $\alpha, \beta$, and $\gamma$. Under physiological conditions, the $\beta$ subunit is strongly associated with the $\gamma$ subunit, which is anchored to plasma membranes by prenylation (Lai et al, 1990). The biochemical natures of the candidate molecules, namely $\mathrm{G} \beta 1$ and $\mathrm{G} \beta 2$, are totally consistent with that of Raft. 1 antigen, ie, their molecular weights are $34 \mathrm{kd}$, their isoelectric points are slightly more basic than that of actin, and they are resistant to detergent.

\section{Confirmation of the Antigen Molecule with GST-Fusion Proteins}

To confirm that Raft. 1 reacts with $G \beta 1$ and $G \beta 2$, recombinant $\mathrm{G} \beta 1$ and $\mathrm{G} \beta 2$ were produced as GST fusion proteins and used for Western blotting analysis. The Triton X-100 extracts from the white and gray matter of a rat brain was used as a source of $\mathrm{G} \beta 5$. Raft. 1 bound to both the GST-G $\beta 1$ and $-\mathrm{G} \beta 2$ fusion proteins but not to rat brain $\mathrm{G} \beta 5$, while $\mathrm{G} 4$ did not bind to either GST-G $\beta 1$ or $-\mathrm{G} \beta 2$ fusion proteins but bound to rat brain $\mathrm{G} \beta 5$. Polyclonal anti-G $\beta$ antibody $\mathrm{T}-20$ bound to all of the $\mathrm{G} \beta \mathrm{s}$ (Fig. 5). Because $\mathrm{G} 4$ was raised against the bovine brain $\mathrm{G} \beta \gamma$ proteins, G4 is thought to recognize the $\mathrm{G} \beta 5$ subunit. The homology between $\mathrm{G} \beta 1$ and $\mathrm{G} \beta 2$ is greater than $90 \%$, but the homology between $\mathrm{G} \beta 1 / 2$ and $\mathrm{G} \beta 5$ is only $52 \%$. These results conclusively demonstrate that Raft. 1 recognizes $\mathrm{G} \beta 1$ and $\mathrm{G} \beta 2$.

\section{Imunoprecipitation with Raft.1 from Raft Microdomains}

The immunoprecipitates with Raft.1 (Fig. 6, lane 1) or with negative control antibody (lane 2) from ACHN raft microdomains were separated by SDS-PAGE and transferred to a nitrocellulose membrane. When the blot was probed with $\mathrm{T}-20$, a clear band appeared at the position of $G \beta$ in lane 1 and was identical to that detected with the crude raft proteins in lane 3 but not in lane 2. This shows that the immune complex with Raft. 1 contains $\mathrm{G} \beta$ and that Raft. 1 can precipitate $\mathrm{G} \beta$ from raft microdomains.

\section{Subcellular Distribution of G及1 and 2 by Sucrose Density Gradient Centrifugation}

Sucrose density gradient centrifugation of $1 \%$ Triton lysate from total cells separated G $\beta 1$ and 2 into a DIM fraction and a detergent-soluble fraction (DS) with a $1: 8$ ratio (Fig. $7, a-i)$. To more precisely analyze the distribution of $\mathrm{G} \beta$, we separated plasma membranes from the microsome fractions and assessed the detergent insolubility of $\mathrm{G} \beta 1$ and 2 in both fractions. The 

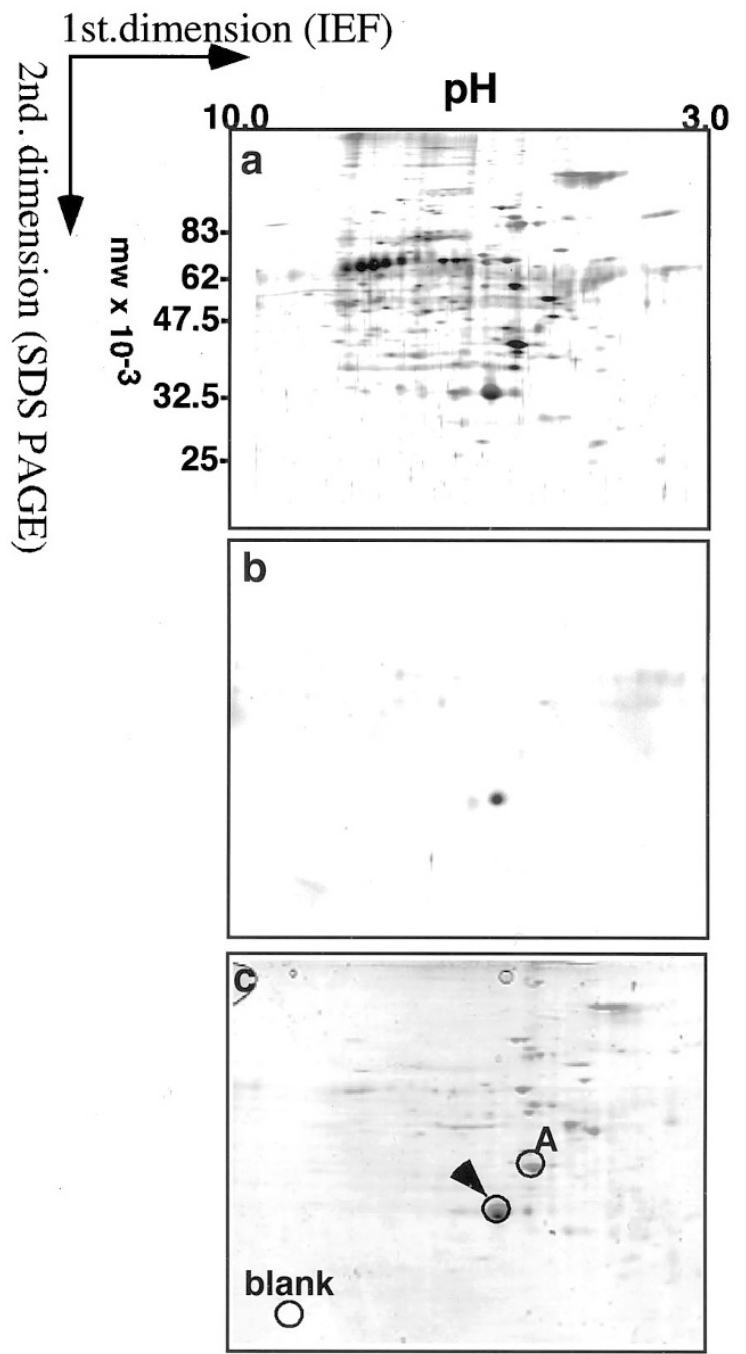

Figure 3.

Antigen analysis of 2-D Western blotting. a, Five micrograms of raft proteins were separated by IEF in the first dimension and then by SDS PAGE in the second dimension. The gel was fixed in $50 \%$ methanol/10\% acetic acid/water and then silver-stained. $\mathrm{b}$, The proteins $(5 \mu \mathrm{g})$ separated as above were transferred to a nitrocellulose membrane and probed with Raft.1. c, Approximately $100 \mu \mathrm{g}$ of raft proteins were separated by 2-D PAGE in the same manner and stained with CBB. Three circles indicate the spots to be subjected to MALDI-TOF MS analysis. The circle indicated by the arrowhead corresponds with the Raft.1 antigen. $A$ indicates actin molecules used as a positive control for the analysis. Blank indicates the gel used for identification of the background peaks.

$\mathrm{G} \beta 1$ and 2 of the plasma membrane were recovered in DI/DS in a 1:2 ratio (Fig. 7, a-ii), whereas they were mostly recovered from other microsome membranes in DS (Fig. 7, a-iii). These indicate that the G $\beta 1$ and 2 in intracellular membranes are detergent sensitive. The 2-D pattern of the DIM proteins of the plasma membrane is shown in Figure $7 \mathrm{~b} . \mathrm{G} \beta 1$ and 2 were the major molecules easily recognized by protein staining in 2-D gel. Because actin was polymerized in the low ionic lysis buffer containing $\mathrm{Ca}^{2+}$, which is required for plasma membrane preparation, more actin appeared under these conditions than in $\mathrm{Ca}^{2+}$-free lysis buffer (compare with Fig. 3a).

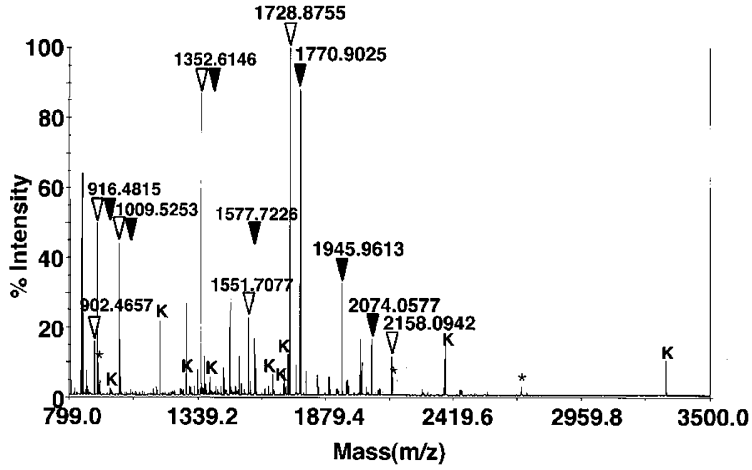

Figure 4.

MALDI-TOF MS spectra of the 34-kd molecules recognized with Raft.1 MAb. The Raft. 1 antigen molecule indicated by the arrowhead in Figure $2 \mathrm{c}$ was in-gel digested with trypsin and subjected to MALDI-TOF MS spectrometry. The mass of peaks marked with the closed and open triangles matched $\mathrm{G} \beta 1$ and $\mathrm{G} \beta 2$, respectively. The $K$ peaks were derived from keratin, and the peak marked ${ }^{*}$ were the same to the spectrum of blank.

Table 1. Mass Fragments Fitted for Human G $\beta 1$ and G $\beta 2$ by MS-fit Search

\begin{tabular}{|c|c|c|c|}
\hline $\mathrm{m} / \mathrm{z}$ & $\begin{array}{c}\mathrm{G} \beta \\
\text { subunit }\end{array}$ & Start End & Peptide sequence \\
\hline 902.4657 & 1 & 1 & 8 SELDQLR \\
\hline 916.4815 & $1,2 \dagger$ & 130138 & 38 EGNVRVSR \\
\hline 1009.5253 & $1,2 \dagger$ & $305 \quad 314$ & 14 AGVLAGHDNR \\
\hline 1352.6146 & $1,2 \dagger$ & $58 \quad 68$ & 68 IYAMHWGTDSR \\
\hline 1551.7077 & 1 & 138150 & 50 ELAGHTGYLSCCR \\
\hline 1577.7226 & 2 & $138 \quad 150$ & 50 ELPGHTGYLSCCR \\
\hline 1728.8755 & 1 & 115 & 15 SELDQLRQEAEQLK \\
\hline 1770.9025 & 2 & 115 & 15 SELEQLRQEAEQLR \\
\hline 1945.9613 & 2 & $24 \quad 42$ & 42 ACGDSTLTQITAGLDPVGR \\
\hline 2074.0577 & 2 & 2342 & 42 KACGDSTLTQITAGLDPVGR \\
\hline 2158.0942 & 1 & 2342 & 42 KACADATLSQITNNIDPVGR \\
\hline
\end{tabular}

$\dagger$ Fragments common to $G \beta 1$ and $G \beta 2$.

\section{Translocation of G及1 and 2 Molecules After Stx Stimulation}

Next, we examined how $\mathrm{G} \beta 1$ and 2 were distributed in unstimulated and Stx-stimulated ACHN cells using a confocal microscope. G $\beta 1$ and 2 were filamentously distributed, radiating in all directions from the vicinity of the nucleus to the periphery of unstimulated ACHN cells (Fig. 8, left row). Once ACHN cells were stimulated with Stx, they accumulated near nucleus (Figure 8, right row). Comparing Stx $-/+$ pairs of panels of Raft.1 staining scanned from a substratum (upper panel) to an apical face (lower panel), it was clearly shown that $\mathrm{G} \beta 1$ and 2 assembled in the vicinity of the nucleus after Stx stimulation.

\section{Immunohistochemical Examination of G及1 and 2 in Rat Tissues}

Tissue sections obtained from male rats were subjected to immunohistochemical staining with Raft.1. Raft. 1 stained virtually all tissues examined, and some representative results are shown in Figure 9a. G $\beta 1$ and 


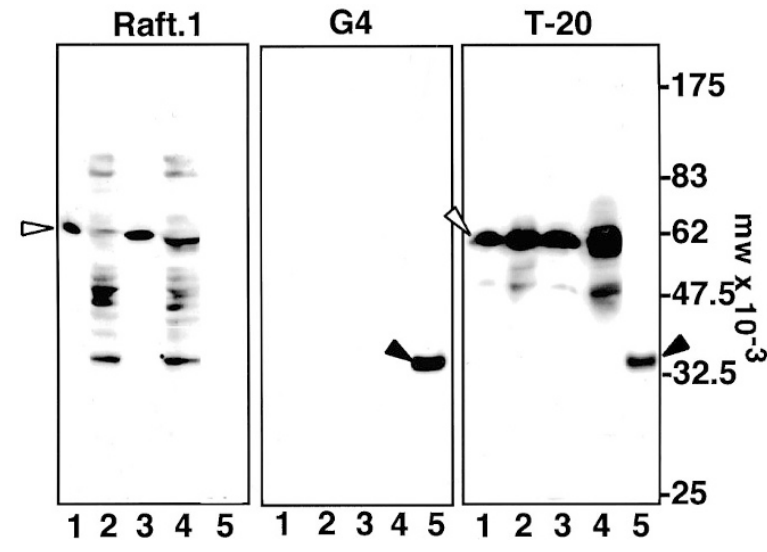

Figure 5.

Confirmation of the antigen molecules with GST-G $\beta 1$ and $\beta 2$ fusion proteins. Purified GST-G $\beta$ fusion protein (lanes 1 and 3, open triangle) and $\mathrm{DH} 5 \alpha$ lysate (lanes 2 and 4 ) were separated by SDS PAGE in a 10\% acrylamide gel and transferred to a nitrocellulose membrane in triplicate. The Triton X-100 extracts from the rat brain were subjected to lane 5 as a source of $G \beta 5$ (closed triang/e). The blot was probed with Raft.1, G4, or T-20. GST-G $\beta 1$ in lanes 1 and 2, GST-G $\beta 2$ in lanes 3 and 4 , rat brain extracts in lane 5 .

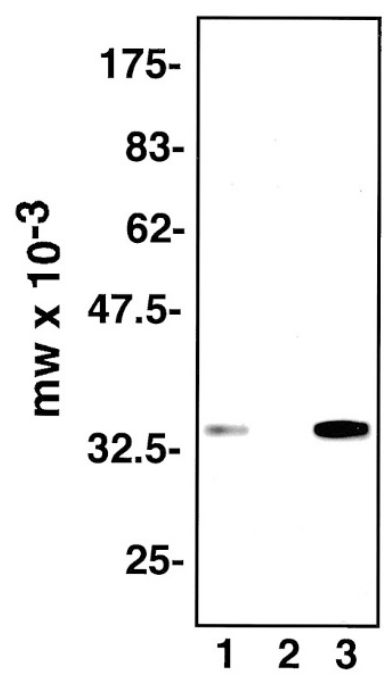

Figure 6.

Immunoprecipitation with Raft.1 from ACHN raft microdomains. The immunoprecipitates with Raft.1 (lane 1) or negative control antibody (lane 2) were examined by Western blotting analysis. The blot was probed with T-20. An aliquot of raft microdomains was loaded in lane 3 as a positive control for T-20 staining.

2 are known as transducin $\beta 1$ and $\beta 2$ and were initially isolated from bovine retina (Fung, 1983). G $\beta 1$ and 2 were clearly and consistently identified in rat retina with Raft.1. Rod and cone outer segments, outer limiting membranes, outer and inner plexiform layers, and ganglion cell layer, all of which are rich in nerve fibers, stained positively. In the testis, the middle piece of sperm and the small spots near the nucleus of spermatids stained. Cardiac muscle also stained, and the staining was superimposed on the cross striations. In the liver, the cell membrane of all hepatocytes stained, and, interestingly, dense staining was obtained in areas of tight contact between neighboring cells. Hair follicles showed prominent immunoreactiv-

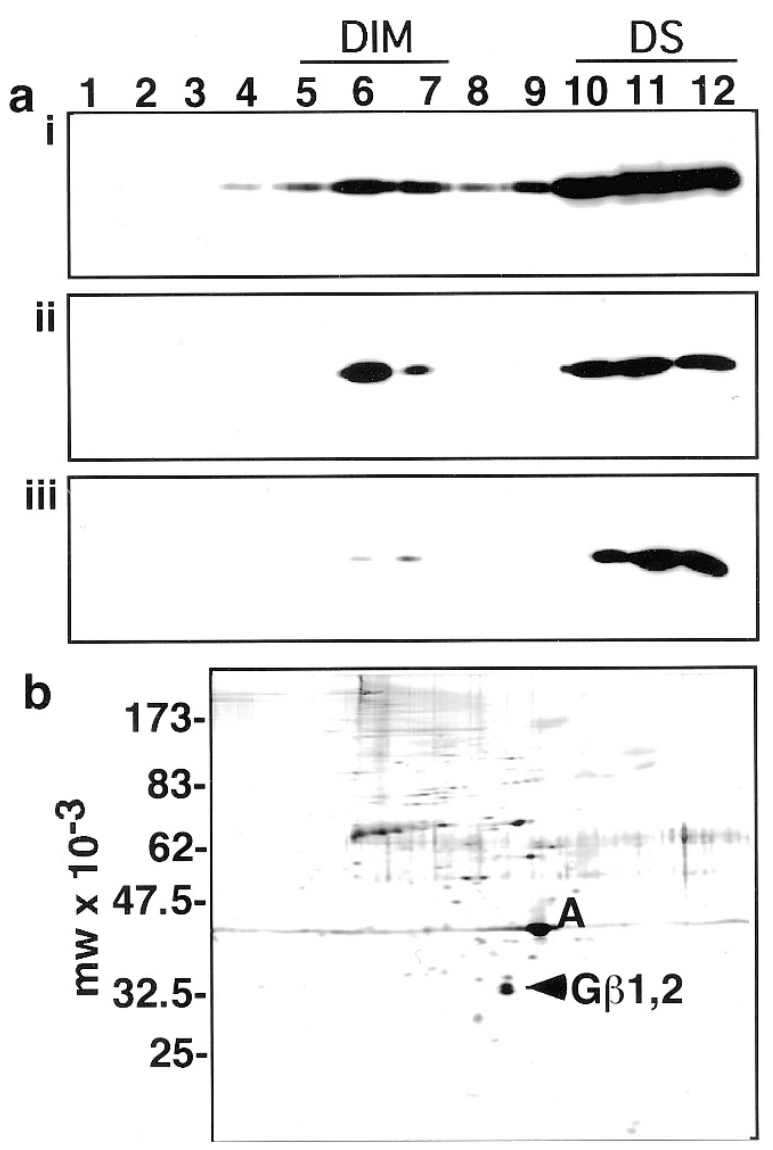

Figure 7.

Subcellular distribution of $\mathrm{G} \beta 1$ and 2 by sucrose density gradient centrifugation analysis. a, Triton lysates from whole cells (i), plasma membranes (ii), and the other microsome fractions (iii) were separated by sucrose density gradient centrifugation. Each fraction was analyzed by Western blotting and probed with T-20. b, Raft proteins prepared from plasma membrane (DIM in ii) were separated by 2-D PAGE and were silver stained.

ity in hair sheaths and hair papillae. In the kidney, mainly the glomeruli and the apical side of some renal tubules stained. Because the brain is known to be rich in $\mathrm{G}$ proteins, we examined rat brain tissue sections for reactivity with Raft.1 and G4 (Fig. 9b). Raft.1 clearly stained the apical side of choroid plexus epithelial cells and ependymal cells (left), but G4 did not and instead stained brain matter rich in nerve fibers (right).

\section{Discussion}

In order to know the role of raft microdomains in signal transduction, many studies have been performed utilizing various techniques. Studies by confocal microscopy have helped to show how raft microdomains assemble (Janes et al, 1999) and how they are internalized after ligand binding (Grakoui et al, 1999). Biochemical approaches have enabled us to characterize raft microdomains chemically (Ahmed et al, 1997). Western blotting has allowed kinetic analyses of signaling molecules, both quantitatively and qualitatively (Iwabuchi et al, 1998b). Use of cholesteroldepleting reagents, such as methyl $\beta$-cyclodextrin, or cholesterol-binding reagents, such as filipin, have 
Stx
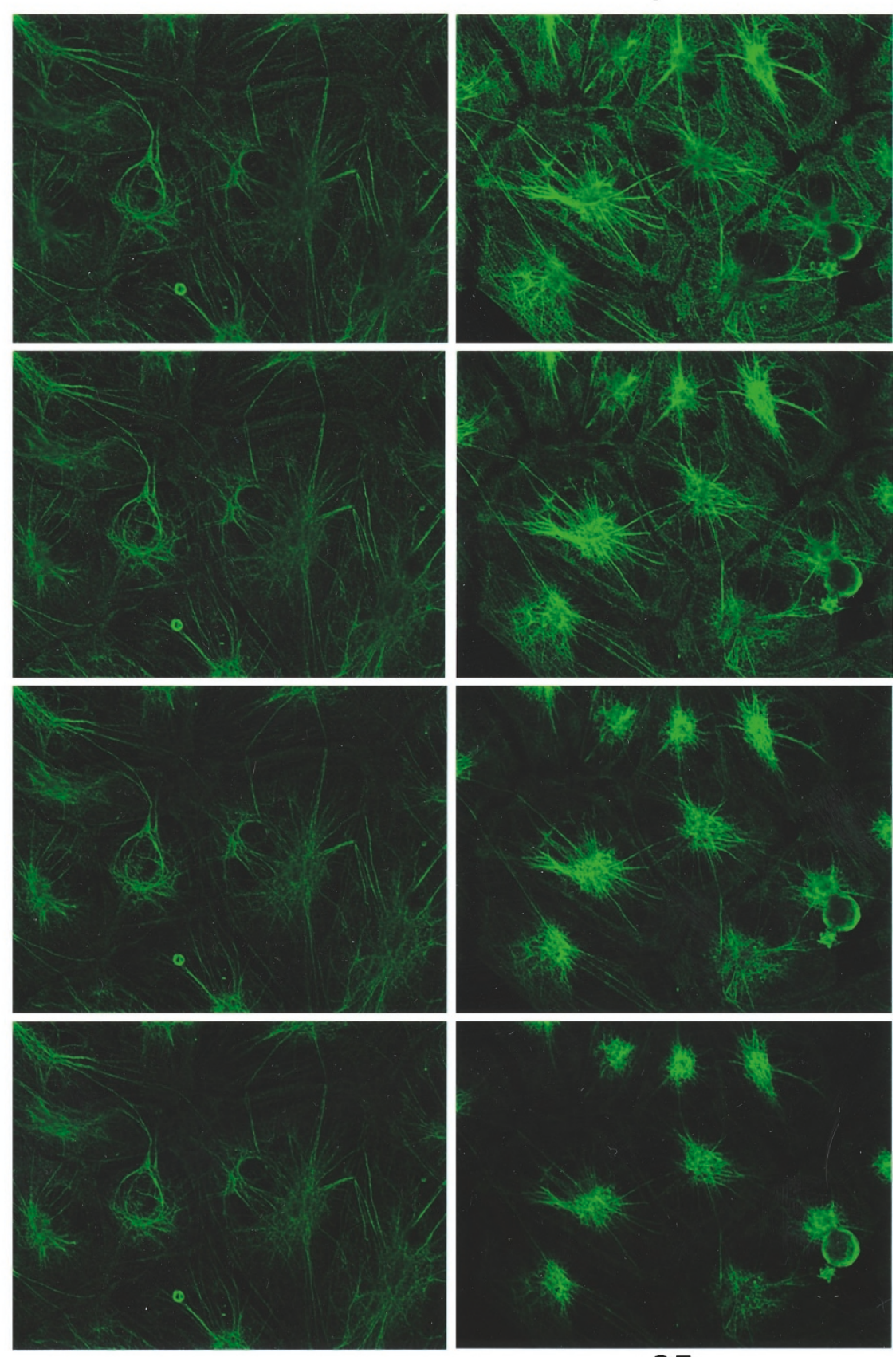

$25 \mu \mathrm{m}$

\section{Figure 8.}

Intracellular localization of $\mathrm{G} \beta 1$ and 2 molecules after Stx stimulation. ACHN cells grown on a collagen-coated cover slip were treated as in Figure 1 and stained with Raft.1. The $\mathrm{G} \beta 1$ and 2 molecules were visualized by indirect immunofluorescence with Raft.1 MAb and Alexa 488-conjugated anti-mouse $\mu$ chain and observed with a confocal microscope. Scanned from a substratum (upper panel) to an apical side (lower panel).

shown the role of cholesterol and the significance of integrating raft structure (Iwabuchi et al, 1998a). Reconstitution of molecules into chemically defined raft microdomains has simplified the system and explained many phenomena (Moffett et al, 2000). We ourselves have studied the early events involved in raft-mediated signal transduction in kidney cells (Sekino et al, 2002) and lymphoid cells (Suzuki et al,
2001) after Stx binding or stimulation with immobilized antibody.

The results of the above have suggested the need to characterize the constituent molecules and determine their exact structure and interrelationships. The great advances have been made in the techniques used to characterize constituent molecules, and they have been applied to the analysis of raft microdomains. 
a.(i)Retina

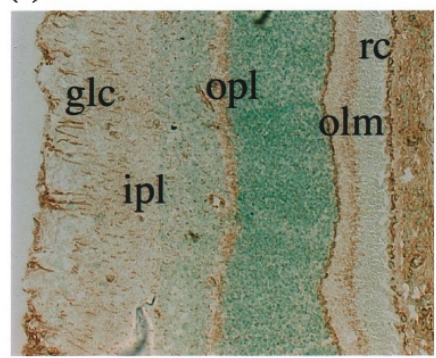

(iv)Liver

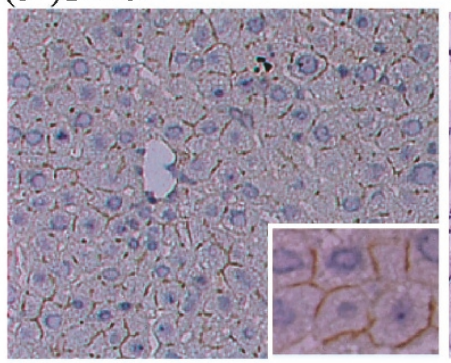

b.Raft.1

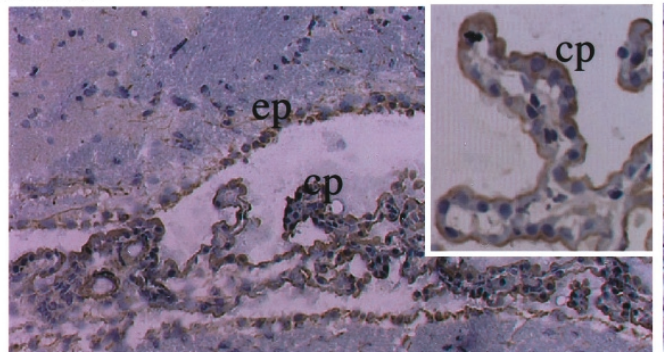

(ii)Testis

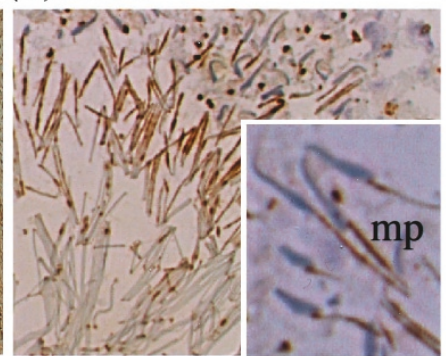

(v)Hair follicle

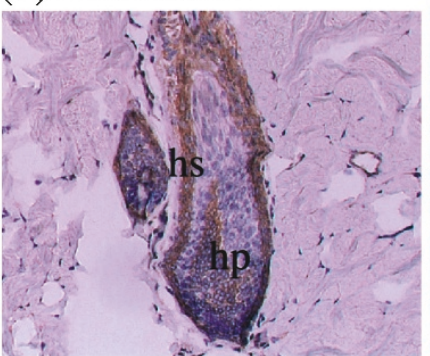

G4
(iii)Heart

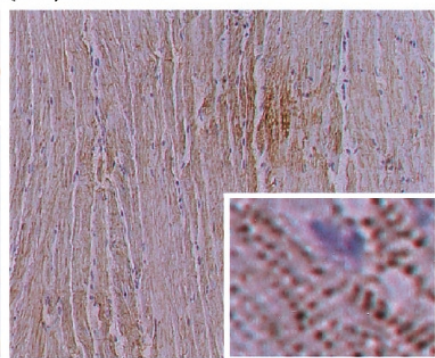

(vi)Kidney

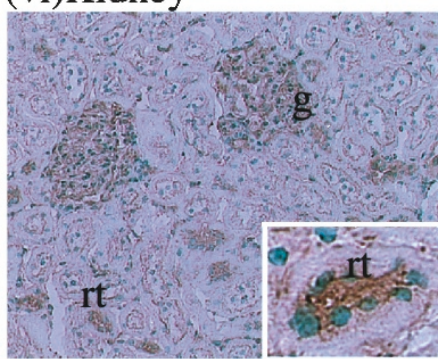

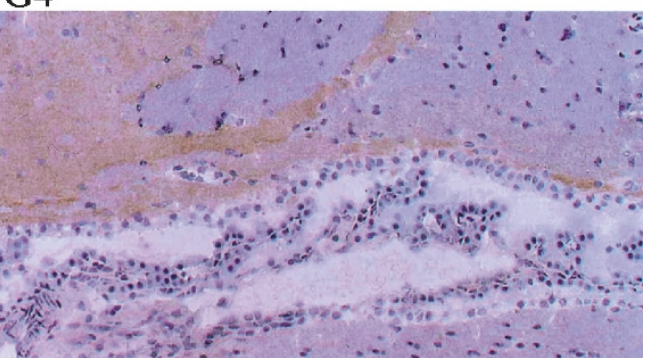

\section{Figure 9.}

Raft.1 immunostaining in various rat organs (a) and comparison of the immunoreactivity and specificity of Raft.1 with G4 (b). a, The frozen sections of retina (i), testis (ii), heart (iii), liver (iv), hair follicle (v), and kidney (vi) obtained from a male rat were fixed in cold acetone and immunostained with Raft.1. (i) was counterstained with methyl green and the others with hematoxylin. The insets in (ii), (iii), (iv), and (vi) show positive immunostaining of the middle piece of sperm, cross striations of heart muscle, adjacent hepatic cells, and renal tubules, respectively. glc, ganglion cells; ipl, inner plexiform layer; opl, outer plexiform layer; olm, outer limiting membrane; rcs, rod and cone segments; $m p$, middle piece; $h s$, hair sheath; $h p$, hair papilla; $g$, glomerulus; $r t$, renal tubule. b, Frozen sections of rat brain were immunostained with Raft.1 (left) or G4 (right) and counterstained with hematoxylin. The inset in the right panel shows the positive staining on the apical side of the choroid plexus. $c p$, choroid plexus; ep, ependymal.

Electrospray ionization mass spectrometry has revealed the composition of phospholipids in raft microdomains and allowed comparison of them with those of nonraft plasma membranes (Pike et al, 2002). Proteins from Jurkat $T$ cell rafts have been characterized by microcapillary liquid chromatography electrospray ionization tandem mass spectrometry (Von Haller et al, 2001), and 70 proteins were identified, most of which were cytoskeletal proteins, such as actin, tubulin, myosin, etc. Lck was identified as the major signaling molecule, and slight but significant amounts of heterotrimeric GTP binding proteins were also identified.

We have approached characterization of raft microdomains by raising MAbs against them with the intention of screening the clones that recognize molecules anchoring to the cytoplasmic face of the raft microdomains and changing their locations with signaling processes. G $\beta 1$ and 2 molecules recognized with Raft.1 are in accord with what we have searched. $\mathrm{G}$ proteins are expressed on the cytoplasmic face of plasma membranes and translocate with signaling processes (Luttrell et al, 1999). We showed that G $\beta 1$ and 2 molecules are filamentously distributed, radiating from the vicinity of a nucleus to the cell periphery, and that they accumulate in the center of radial filaments after Stx stimulation. A direct interaction between $\mathrm{G} \beta \gamma$ and tubulin was suggested in neuronal signal transduction (Roychowdhury and Rasenick, 1994). However, our preliminary confocal microscopic observation showed that the filamentous staining pattern produced by Raft.1 did not overlap with the actin filaments but partially overlapped with vimentin. Some GSLs are reported to be associated with the vimentin intermediate filaments of the human umbilical vein endothelial cells (Gillard et al, 1991). The staining patterns of glycosphingolipids such as MSGG and Gb3 of ACHN cells completely differed from that of $\mathrm{G} \beta 1$ and 2 (data not shown). Evanko et al (2001) demonstrated that, when myc-tagged $\mathrm{G} \beta 1 \sim 4$ subunits were coexpressed with $\mathrm{G} \gamma 3$ subunits in HEK293 cells, $\mathrm{G} \beta \gamma$ subunits displayed cytoplasmic localization and bright intracellular aggregates. This bright intra- 
cellular aggregate may be possibly equal to the site where $\mathrm{G} \beta 1$ and 2 subunits assembled in Stx-stimulated ACHN cells. What the filamentous structure bound by Raft.1 is remains unclear. In lymphoid cell lines, Raft.1 exclusively stained plasma membrane (data not shown).

Heterotrimeric G proteins serve as central signaling molecules responsible for connecting cellular signals transduced from receptors to their respective effectors, and the exchange of GDP for GTP in the $\alpha$ subunits results in dissociation of the $\mathrm{G} \alpha$ subunits from the $\mathrm{G} \beta \gamma$ subunits (Wall et al, 1995). Caveolin is a major structural component of caveolae and has been found to interact with $\mathrm{G}$ proteins and various downstream effector molecules (Oka et al, 1997; Toya et al, 1998), and agonist-stimulation has been shown to promote sequestration of $\mathrm{G} \alpha$ into the detergentinsoluble caveolin-rich fractions (de Weerd and LeebLundberg, 1997). We also found that caveolin is coimmunoprecipitated with $\mathrm{G} \beta 1$ and 2 of $\mathrm{ACHN}$ raft proteins (data not shown). Chronic receptor activation has recently been shown to lead to a decrease in the detergent solubility of $\mathrm{G} \alpha$ and $\mathrm{G} \beta \gamma$ without changing their total contents (Bayewitch et al, 2000), and these studies indicate that $\mathrm{G} \alpha$ or $\mathrm{G} \beta \gamma$ shift location from rafts to nonraft after agonist stimulation. We recovered $\mathrm{G} \beta$ both in the DIM fraction (raft) and the DS fraction (nonraft) of plasma membrane, but almost all of it was in the DS fraction of the other microsome fractions, suggesting that the $\mathrm{G} \beta$ found near the nucleus is probably detergent soluble. Raft.1 can be used to study the cellular localization of $\mathrm{G}$ proteins to determine the fate of $\mathrm{G} \beta$ after agonist stimulation.

During the process of MAb production, raft microdomains themselves were shown to be efficient immunogens. Molecules tightly anchored to raft microdomains, such as G $\beta$ and MSSG, can act as immunogens with no need of adjuvants. Furthermore, because $\mathrm{G} \beta 1$ through 4 is highly conserved and widely distributed in various tissues, production of the MAb was expected to be difficult, but using raft microdomains as immunogens solved the problem. This is the first report of the production of a MAb that recognizes $\mathrm{G} \beta 1$ and $\mathrm{G} \beta 2$. Of particular interest, Raft.1 reacts not only with human cells but with cells from other species, such as rat and pig, as shown in our study. It is therefore likely that the epitope recognized by Raft. 1 resides in the sequence that is shared among species. $\mathrm{G} \beta 5$ is preferentially expressed in neural tissues, such as brain (Watson et al, 1994), and it is possible to purify $\mathrm{G} \beta 5$ from brain in sufficient quantity to produce MAbs. Chiba et al (1995) produced MAb G4 against $\mathrm{G} \beta$ by immunizing mice with $\mathrm{G} \beta \gamma$ purified from bovine brain. Our comparative immunostaining with Raft.1 and G4 suggests that the antigen molecule of $\mathrm{G} 4$ may be $\mathrm{G} \beta 5$. Raft. 1 heavily stained choroid plexus in rat brain but not the brain matter itself, whereas G4 staining was the opposite. Various types of $\mathrm{G}$ proteins have been reported to be expressed in cultured cerebral endothelial cells (Fabian et al, 1998). Raft.1 can be thought to react with $\mathrm{G} \beta 3$ and $\mathrm{G} \beta 4$ in addition to $\mathrm{G} \beta 1$ and $\mathrm{G} \beta 2$. This is an advantage for staining $\mathrm{G}$ proteins other than $\mathrm{G} \alpha \times \beta 5$ as a whole. The immunohistochemical study of acetonefixed frozen sections of rat tissues gives us interesting information. $\mathrm{G} \beta$ is expressed abundantly in junctions between adjacent liver cells where bile is secreted or on the apical side of epithelial cells through which body fluids pass. Athe $\mathrm{CHN}$ cells, which have been derived from renal epithelial cells, are an example of the latter. $\mathrm{G} \beta$ also abounds in the middle piece of sperm and the cross striations of heart muscle, where large amounts of ATP are consumed, and in the papillae of hair follicles, in which hairs are actively synthesized. Thus, Raft.1 MAb can be expected to be useful as a probe for research not only in regard to cellular location but morphogenesis as well.

\section{Materials and Methods}

\section{Cell Culture, Antibodies, and Chemical Reagents}

The ACHN cells, a human renal tubular carcinoma cell line, were purchased from the American Type Culture Collection and were maintained in Eagle's MEM (Sigma Chemical Company, St. Louis, Missouri) supplemented with nonessential amino acids supplements (Gibco Laboratories, Grand Island, New York) and $10 \%$ fetal bovine serum (Sigma). Nalm 20 cells (pro-B cell line), HPB-NULL cells (pre-B cell line), Ramos cells (Burkitt's-derived B cell line), ER cells (EB-virus transformed $B$ cell line), and IM-9 cells (IgG-secreting myeloma) were obtained from the Japanese Cancer Research Resources Bank and were cultured in RPMI (Sigma) medium supplemented with $10 \%$ fetal bovine serum. P3U1 cells, a mouse myeloma cell line obtained from the American Type Culture Collection, were maintained in RPMI medium supplemented with $10 \%$ fetal bovine serum. Raft.2, anti-MSGG MAb, has been described previously (Katagiri et al, 2001). Anti-Yes MAb was purchased from Transduction Laboratories, Lexington, Kentucky. G4 (Chiba et al, 1995), a MAb for bovine brain $\mathrm{G}$ protein $\beta$ subunit, was kindly donated by $\mathrm{Dr}$. Chiba of Ochanomizu University, Tokyo, Japan. G $\beta$ (T-20), a rabbit anti-mouse $\mathrm{G} \beta 1$ polyclonal antibody, was purchased from Santa Cruz Biotechnology (Santa Cruz, California). The isotype of the cloned MAb was determined by using MAb-based mouse lg isotyping kit (PharMingen, San Diego, California).

\section{Preparation of Raft Microdomains}

Raft microdomains were prepared as described by Katagiri et al (2001). Briefly, confluent cultures of ACHN cells in a 10-cm culture plate were lysed in 0.7 $\mathrm{ml}$ of Tris-buffered saline $(25 \mathrm{~mm}$ Tris- $\mathrm{HCl}$ buffer, $\mathrm{pH}$ 7.5/0.15 M NaCl) containing 1\% Triton $\mathrm{X}-100,1 \mathrm{~mm}$ phenylmethylsulfonyl fluoride, $1 \mathrm{mM} \mathrm{Na} \mathrm{NO}_{4}$, and aprotinin (10 trypsin inhibitor units $/ \mathrm{ml}$ ), and the pellet was homogenized in a Teflon glass homogenizer on ice. Cell extracts were adjusted to $40 \%$ sucrose in Tris-buffered saline and placed in a 12-ml ultracentrifugation tube. A discontinuous 5\%/30\% sucrose gradient was formed above the sample. Raft microdomains were obtained as DIM at the interface between the 5 and $30 \%$ sucrose layers after ultracentrifugation 
for 16 hours at $39,000 \mathrm{rpm}$ at $4^{\circ} \mathrm{C}$ in a Beckman SW 40Ti rotor.

Alternatively, raft microdomains were obtained from membrane fractions prepared according to the method of Forte et al (1973) with slight modification. The ACHN cells were homogenized in hypotonic buffer $\left(25 \mathrm{~mm} \mathrm{NaCl}, 0.5 \mathrm{~mm} \mathrm{CaCl}_{2}, 18 \mathrm{~mm}\right.$ Tris $\mathrm{HCl}$ buffer, $\mathrm{pH} 8.0$, and protease inhibitors) with a Teflon glass homogenizer and centrifuged at $200 \times g$ for 1 minute. Precipitates were homogenized in the same manner twice more. Ultracentrifugation of the combined supernatants at $40,000 \mathrm{rpm}$ for 30 minutes in a Beckman 80Ti rotor precipitated microsome fractions, and they were thoroughly suspended in hypotonic buffer containing 30\% sucrose and laid over a discontinuous sucrose gradient composed of $40 \% / 45 \% /$ $50 \% / 60 \%$ in a $12-\mathrm{ml}$ ultracentrifugation tube. Plasma membrane fractions were recovered at the interphase between the 40 and $45 \%$ sucrose layer after centrifugation at 25,000 rpm for 1 hour in a SW40Ti rotor. Plasma membranes thus obtained were lysed in Trisbuffered saline containing 1\% Triton X-100 and were separated by a sucrose density gradient centrifugation as described above.

\section{Production of MAbs}

MAbs were produced as previously described (Katagiri et al, 2001). Briefly, a PBS suspension of raft microdomains (4.85 $\mu \mathrm{g}$ protein per one injection) was subcutaneously injected into 6-week-old Balb/c female mice, and after several booster shots, the spleen cells were fused with the P3U1 mouse myeloma cell line by using $38 \%$ polyethylene glycol HYBRI-MAX (Sigma). Hybrid cells were selected in S-clone cloning medium CM-B (Sanko-Jyunyaku, Tokyo, Japan) containing hypoxanthin, aminopterine, and thymidine (Sigma). The culture supernatant of the hybrid cells was screened first by dot-blot immunostaining assay and then by flowcytometric analysis. The hybridoma clones of which culture supernatants were positive for raft microdomains were selected and further subcloned by the limiting dilution method.

\section{Staining for Fluorescence Microscopic Observation}

$\mathrm{ACHN}$ cultures in RS-coated glass chamber slides (Nalge Nunc International, Naperville, Illinois) or on collagen-coated cover slips were incubated in the absence or the presence of Stx $(10 \mathrm{ng} / \mathrm{ml})$ for 30 minutes and fixed with ice-cold acetone for 15 minutes. The immunostaining procedure included adequate hydration with PBS for 15 minutes and blocking with $5 \%$ nonimmunized rabbit or goat serum for 30 minutes. The slides were incubated with a primary antibody at room temperature for 1 hour, then FITCconjugated rabbit anti-mouse IgG $+\mathrm{M}$ antibody (Jackson ImmunoResearch, West Grove, Pennsylvania) or Alexa Fluor 488-conjugated goat anti-mouse IgM ( $\mu$ chain) (Molecular Probes, Eugene, Oregon) for 30 minutes. The slides were mounted with Perma Fluor Aqueous Mounting Medium (Thermo Shandon, Pitts- burgh, Pennsylvania) and observed using a conventional fluorescence microscope or an Olympus LSMGB200 confocal microscope.

\section{Flowcytometric Analysis}

Cells were harvested and incubated with Raft. 1 before and after treatment with Cytofix/Cytoperm (PharMingen). They were then stained with FITC-conjugated goat anti-mouse $\lg G+M$ antibody (Jackson) and analyzed with a flowcytometer (EPICS-XL; Beckman Coulter). Peripheral blood cells obtained from a human volunteer, pig, and rat were exposed to the hemolysis buffer and then stained similarly.

\section{PAGE and Immunoblotting}

Proteins were separated on $10 \%$ polyacrylamide gels under reduced conditions after dissolving in loading buffer containing 1.7\% SDS and $0.1 \mathrm{M}$ dithiothreitol. Two-dimensional PAGE was performed using the Mini-PROTEAN II 2-D system (Bio-Rad Laboratories, Hercules, California) according to the manufacturer's instructions. Proteins were separated in the first dimension by isoelectric focusing in capillary tubes with a $\mathrm{pH}$ range of 3.5-10 and in the second dimension by SDS-PAGE as described above.

Separated proteins were transferred to nitrocellulose membranes (Millipore Corporation, Bedford, Massachusetts). The blots were blocked with PBS containing $5 \%$ skimmed milk and incubated with the first antibody and then with horseradish peroxidaseconjugated goat anti-mouse $\operatorname{lgG}+\mathrm{M}$ or goat antirabbit immunoglobulin (Jackson ImmunoResearch). Bands were visualized by enhanced chemiluminescence (ECL Western Blotting System; Amersham Pharmacia Biotech, Buckinghamshire, United Kingdom). Gels were examined for proteins by staining with CBB or with 2-D SILVER STAIN II DAIICHI (Daiichi Pure Chemicals, Tokyo, Japan).

\section{MALDI-TOF MS Analysis}

The raft proteins were separated by 2-D PAGE and stained with CBB. The stained protein spot that corresponded to the position of the Raft.1-reacting spot was excised and digested with trypsin, and the trypsinized peptides were detected with a MALDI-TOF MS spectrometer equipped with delayed ion extraction (Voyager Biospectrometry Workstation DE-STR; Applied Biosystems). The mass spectra were analyzed by an MS-fit database search, and identification was confirmed by postsource decay matrix-assisted laser desorption/ionization mass spectrometry.

\section{Bacterial Expression and Purification of GST Fusion Proteins}

The cDNAs encoding human $\mathrm{G} \beta 1$ and $\mathrm{G} \beta 2$ were amplified by PCR from ACHN cDNA libraries. The primers for G $\beta 1$ were 5'-G $\beta 1$ (5'-AGCACTAAGATCGGAAGATG) and $3^{\prime}-\mathrm{G} \beta 1$ (AGTTCCAGATCTTGAGGAAG-3'). The primers for $\mathrm{G} \beta 2$ were $5^{\prime}-\mathrm{G} \beta 2$ (5'-ATGAGTGAGC- 
TGGAGCAACTGAG) and 3'-GB2 (AGTTCCAGATCTTGAGGAAG-3'). The $5^{\prime}$ primers contained an initiation codon (underlined). The PCR products were cloned into a pGEM-T vector (Promega Corporation), and their sequences were confirmed with an automated fluorescence dye DNA sequencer (ABI Prism 310; Applied Biosystems). To insert the cDNA into pGEX-6P-1 vector (Amersham Pharmacia Biotech), PCR was conducted using pGEM-T vector containing $\mathrm{G} \beta 1$ or $\mathrm{G} \beta 2 \mathrm{cDNAs}$ as a template directed by pairs of primers: $5^{\prime}-\mathrm{G} \beta 1,5^{\prime}-$ TAAGATGAATTCATGAGTGAGCTTGACCAGTTA and 3'-G $\beta 1$, CCGCGGCTCGAGTTCCAGATCTTGAGGAAG-3'; 5'-Gß2, 5'-GGCCGCGAATTCATGAGTGAGCTGGAGCAACT and 3'-Gß2, ACTAGTCTCGAGTTCCAGATCTTGAGGAAG-3'. They contained an EcoRI site (underlined) or a Xhol site (italicized). The EcoRI/Xhol fragments of the PCR products were cloned into a pGEX-6P-1 vector. The Escherichia coli $\mathrm{DH} 5 \alpha$ was transformed with pGEX-6P-1-containing recombinant $\mathrm{G} \beta 1$ or $\mathrm{G} \beta 2$ cDNAs, and the GST fusion proteins were then induced with $1 \mathrm{~mm}$ isopropyl-1-thio- $\beta$-Dgalactopyranoside. The GST fusion proteins were purified with glutathione-conjugated Sepharose beads (Amersham Pharmacia Biotech).

\section{Immunoprecipitation}

Microdomains prepared from two $10-\mathrm{cm}$ culture plates were mixed with Raft. $1 \mathrm{MAb}$ overnight at $4^{\circ} \mathrm{C}$. After thoroughly washing the rafts with PBS containing $1 \%$ triton $X-100$ with a microcentrifuge at $15,000 \mathrm{rpm}$ for 10 minutes, they were rotated with protein A-Agarose (Roche Molecular Biochemicals) to which goat anti-mouse IgG+M antibodies (Jackson ImmunoResearch) had been bound. The bound proteins were separated by SDS-PAGE and transferred to a nitrocellulose membrane as described above. The blot was probed with $G \beta(T-20)$, then with horseradish peroxidase-conjugated goat anti-rabbit immunoglobulin, and finally by visualization with the ECL Western blotting system.

\section{Immunohistochemical Staining with Anti-Gß Antibody}

The frozen sections of brain, eyeball, testis, kidney, heart, skin, and liver from a male Lewis rat were fixed in ice-cold acetone for 15 minutes. The slides were stained as described above. Horseradish peroxidaseconjugated goat anti-mouse $\lg \mathrm{G}+\mathrm{M}$ antibody (Jackson) and diaminobenzidine (DAKO) were used as the second antibody and the chromogen, respectively. All slides were counterstained with hematoxylin or methyl green.

\section{Acknowledgements}

We thank Drs. K. Chiba and M. Hoshi for the kind gift of G4 MAb and also the technical staffs of Applied Biosystems Japan Ltd. for mass analysis.

\section{References}

Ahmed SN, Brown DA, and London E (1997). On the origin of sphingolipid/cholesterol-rich detergent-insoluble cell membranes: Physiological concentrations of cholesterol and sphingolipid induce formation of a detergent-insoluble, liquid-ordered lipid phase in model membranes. Biochemistry 36:10944-10953.

Bayewitch ML, Nevo I, Avidor-Reiss T, Levy R, Simonds WF, and Vogel $Z$ (2000). Alterations in detergent solubility of heterotrimeric $G$ proteins after chronic activation of $\mathrm{G}(\mathrm{i} / \mathrm{o})$ coupled receptors: Changes in detergent solubility are in correlation with onset of adenylyl cyclase superactivation. Mol Pharmacol 57:820-825.

Chiba K, Longo FJ, Kontani K, Katada T, and Hoshi M (1995). A periodic network of $G$ protein beta gamma subunit coexisting with cytokeratin filament in starfish oocytes. Dev Biol 169:415-420.

de Weerd WF and Leeb-Lundberg LM (1997). Bradykinin sequesters B2 bradykinin receptors and the receptorcoupled Galpha subunits Galphaq and Galphai in caveolae in DDT1 MF-2 smooth muscle cells. J Biol Chem 272:1785817866.

Evanko DS, Thiyagarajan MM, Siderovski DP, and Wedegaertner PB (2001). Gbeta gamma isoforms selectively rescue plasma membrane localization and palmitoylation of mutant Galphas and Galphaq. J Biol Chem 276:2394523953.

Fabian G, Szabo CA, Bozo B, Greenwood J, Adamson P, Deli MA, Joo F, Krizbai IA, and Szucs M (1998). Expression of G-protein subtypes in cultured cerebral endothelial cells. Neurochem Int 33:179-185.

Forte JG, Forte TM, and Heinz E (1973). Isolation of plasma membranes from Ehrlich ascites tumor cells. Influence of amino acids on $\left(\mathrm{Na}^{+}+\mathrm{K}^{+}\right)$-ATPase and $\mathrm{K}^{+}$-stimulated phosphatase. Biochim Biophys Acta 298:827-841.

Fung BK (1983). Characterization of transducin from bovine retinal rod outer segments. I. Separation and reconstitution of the subunits. J Biol Chem 258:10495-10502.

Gillard BK, Heath JP, Thurmon LT, and Marcus DM (1991). Association of glycosphingolipids with intermediate filaments of human umbilical vein endothelial cells. Exp Cell Res 192:433-444.

Grakoui A, Bromley SK, Sumen C, Davis MM, Shaw AS, Allen PM, and Dustin ML (1999). The immunological synapse: A molecular machine controlling $T$ cell activation. Science 285:221-227.

Iwabuchi K, Handa K, and Hakomori S (1998a). Separation of "glycosphingolipid signaling domain" from caveolincontaining membrane fraction in mouse melanoma B16 cells and its role in cell adhesion coupled with signaling. $J$ Biol Chem 273:33766-33773.

Iwabuchi K, Yamamura S, Prinetti A, Handa K, and Hakomori $S$ (1998b). GM3-enriched microdomain involved in cell adhesion and signal transduction through carbohydratecarbohydrate interaction in mouse melanoma B16 cells. J Biol Chem 273:9130-9138.

Janes PW, Ley SC, and Magee Al (1999). Aggregation of lipid rafts accompanies signaling via the $\mathrm{T}$ cell antigen receptor. J Cell Biol 147:447-461.

Katagiri YU, Mori T, Nakajima H, Katagiri C, Taguchi T, Takeda T, Kiyokawa N, and Fujimoto J (1999). Activation of Src family kinase Yes induced by Shiga toxin binding to globotriaosyl ceramide (Gb3/CD77) in low density, 
detergent-insoluble microdomains. J Biol Chem 274:3527835282.

Katagiri YU, Ohmi K, Katagiri C, Sekino T, Nakajima H, Ebata T, Kiyokawa N, and Fujimoto J (2001). Prominent immunogenicity of monosialosyl galactosylgloboside, carrying a stage-specific embryonic antigen-4 (SSEA-4) epitope in the ACHN human renal tubular cell line-a simple method for producing monoclonal antibodies against detergentinsoluble microdomains/raft. Glycoconj J 18:347-353.

Lai RK, Perez-Sala D, Canada FJ, and Rando RR (1990). The gamma subunit of transducin is farnesylated. Proc Natl Acad Sci USA 87:7673-7677.

Lingwood CA (1996). Role of verotoxin receptors in pathogenesis. Trends Microbiol 4:145-153.

Luttrell LM, Daaka Y, and Lefkowitz RC (1999). Regulation of tyrosine kinase cascades by G-protein-coupled receptors. Curr Opin Cell Biol Mem 177-184.

Moffett S, Brown DA, and Linder ME (2000). Lipid-dependent targeting of $G$ proteins into rafts. J Biol Chem 275:21912198.

Oka N, Yamamoto M, Schwencke C, Kawabe J, Ebina T, Ohno S, Couet J, Lisanti MP, and Ishikawa Y (1997). Caveolin interaction with protein kinase $C$. Isoenzyme-dependent regulation of kinase activity by the caveolin scaffolding domain peptide. J Biol Chem 272:33416-33421.

Pike LJ, Han X, Chung KN, and Gross RW (2002). Lipid rafts are enriched in arachidonic acid and plasmenylethanolamine and their composition is independent of caveolin-1 expression: A quantitative electrospray ionization/mass spectrometric analysis. Biochemistry 41:2075-2088.

Roychowdhury S and Rasenick MM (1994). Tubulin-G protein association stabilizes GTP binding and activates GTPase: Cytoskeletal participation in neuronal signal transduction. Biochemistry 33:9800-9805.
Sekino T, Kiyokawa N, Taguchi T, Ohmi K, Nakajima H, Suzuki T, Furukawa S, Nakao H, Takeda T, and Fujimoto J (2002). Inhibition of Shiga toxin cytotoxicity in human renal cortical epithelial cells by nitrobenzylthioinosine. J Infect Dis 185:785-796.

Simons K and Ikonen E (1997). Functional rafts in cell membranes. Nature 387:569-572.

Suzuki T, Kiyokawa N, Taguch T, Sekino T, Katagiri YU, and Fujimoto J (2001). CD24 induces apoptosis in human B cells via the glycolipid-enriched membrane domains/raftsmediated signaling system. J Immunol 166:5567-5577.

Taga S, Carlier K, Mishal Z, Capoulade C, Mangeney M, Lecluse Y, Coulaud D, Tetaud C, Pritchard LL, Tursz T, and Wiels J (1997). Intracellular signaling events in CD77mediated apoptosis of Burkitt's lymphoma cells. Blood 90: 2757-2767.

Toya Y, Schwencke C, Couet J, Lisanti MP, and Ishikawa Y (1998). Inhibition of adenylyl cyclase by caveolin peptides. Endocrinology 139:2025-2031.

Von Haller PD, Donohoe S, Goodlett D, Aebersold R, and Watts JD (2001). Mass spectrometric characterization of proteins extracted from Jurkat $\mathrm{T}$ cell detergent-resistant membrane domains. Proteomics 1:1010-1021.

Wall MA, Coleman DE, Lee E, Iniguez-Lluhi JA, Posner BA, Gilman AG, and Sprang SR (1995). The structure of the G protein heterotrimer $\mathrm{Gi}$ alpha 1 beta 1 gamma 2. Cell 83: 1047-1058.

Watson AJ, Katz A, and Simon MI (1994). A fifth member of the mammalian G-protein beta-subunit family. Expression in brain and activation of the beta 2 isotype of phospholipase $\mathrm{C}$. J Biol Chem 269:22150-22156. 